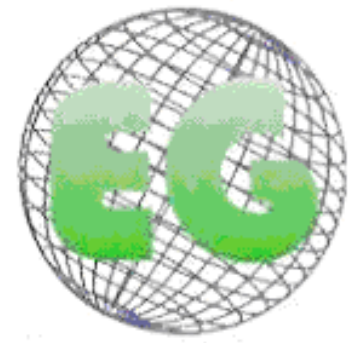

ISSN 1695-6141

\title{
Medidas preventivas para evitar complicaciones de salud derivadas del uso de sondajes vesicales en pacientes lesionados medulares
}

Preventive Measures to Avoid Health Complications from the use of catheters in patients with Spinal Cord Injury

\section{*Torres Alaminos, $\mathrm{M}^{\mathrm{a}}$ Angustias}

\author{
*Grado en Enfermería. Hospital Nacional de Parapléjicos. Toledo. E-mail: angustiast@gmail.com \\ Palabras clave: Paraplejia; Incontinencia urinaria; Evaluación en enfermería; Catéter de permanencia; \\ Bacteriuria.
}

Keywords: Paraplegia; Urinary incontinence; Nursing assessment; Permanent Catheter; Bacteriuria.

\section{RESUMEN}

Introducción: La lesión medular es uno de los sucesos más trágicos que puede acaecerle a una persona por las devastadoras consecuencias que conlleva la parálisis de los 2 ó 4 miembros en forma de paraplejía o tetraplejía, pérdida de las sensibilidades, disfunción vesical, intestinal, sexual y consecuentemente trascendentales secuelas psicológicas, sociales, laborales y económicas.

Objetivos: El objetivo principal es determinar si ciertos tipos de sondas y técnicas de colocación de la sonda son mejores para la prevención de complicaciones a largo plazo en la incontinencia urinaria.

Metodología: La estrategias de búsqueda para la identificación de los artículos de esta revisión los buscamos en bases de datos como Cinahl, Pubmed, Biblioteca Virtual de la Salud, Csic, Biblioteca Cochrane Plus. Las búsquedas se realizaron mediante el sistema de palabras clave. Se llevó a cabo la búsqueda mediante los descriptores $\mathrm{DeCS} / \mathrm{MeSH}$ y además una búsqueda manual de artículos en revistas especializadas de Enfermería tanto españolas como extranjeras.

Resultados: No hay estudios definitivos que revelen que la incidencia de la infección urinaria mejore con alguna técnica, estrategia o tipo de sonda; la dificultad de estos estudios consiste pues en que se necesita un periodo largo de tiempo y muchos pacientes abandonan el estudio.

Conclusiones: Los datos disponibles sobre el sondaje intermitente no aportan pruebas convincentes de que la técnica especifica (estéril o limpia), tipo de sonda (recubierta o no recubierta); método (único uso o uso múltiple), personas (paciente u otro), o estrategia sea mejor que otra para todos los contextos clínicos. Las pruebas actuales son débiles y se recomiendan estudios bien diseñados. Valorar el impacto en la calidad de vida, supone la utilización de distintos métodos de evacuación urinaria en pacientes con lesión medular, utilizando un cuestionario específico y validado al español: King's Health Questionnaire 


\section{ABSTRACT}

Introduction: Spinal cord injury is one of the most tragic events that can befall a person for the devastating consequences associated with paralysis of 2 or 4 members in the form of paraplegia or quadriplegia, loss of sensitivity, bladder dysfunction, bowel, sexual and consequently transcendental psychologic social, labor and economical consequencies.

Objectives: Main objective is determining if certain types of probes and catheterization techniques are the best for preventing long-term complications in urinary incontinence.

Methodology: Search strategies for identifying articles of this review include databases such as Cinahl, Pubmed, Virtual Health Library, CSIC, and Cochrane Library. Searches were conducted using the keyword system: We carried out a search using Mesh descriptors / Mesh and a manual search of articles in Nursing journals from Spain and foreign countries.

Results: There are no definitive studies that reveal that the incidence of UTI improves with some kind of technique, strategy or probe type. The main difficulty for these studies is the long duration; many patients leave the study.

Conclusions: The available data on intermittent catheterization does not provide convincing evidence for the specific technique (sterile or clean), probe type (coated or uncoated), method (single use or multiple use), people (patient or other), or strategy is better than another for all clinical settings. The current evidence is uninformative and well-designed studies are recommended. Evaluating the impact on quality of life involves the use of different methods of evacuation in patients with spinal cord injury using a specific questionnaire validated in Spanish: King's Health Questionnaire

\section{INTRODUCCIÓN}

La lesión de la médula espinal afecta a la regulación de los órganos por debajo del nivel de la lesión, creando un amplio espectro de problemas de salud que pueden variar según la cronicidad del proceso y además alterar los signos y síntomas que pueden provocar una complicación.

Las complicaciones a largo plazo van desde complicaciones del aparato locomotor (problemas posturales en la silla de ruedas, contracturas que pueden llevar a la anquilosis, osificaciones para-articulares, fracturas patológicas debidas a la osteoporosis por inmovilidad), espasticidad (con el aumento desordenado de los reflejos tónicos de estiramientos en relación a la hiperreflexia tendinosa), úlceras por presión (provocadas por la inmovilidad, ausencia del dolor y disminución de tejidos blandos), disreflexia autonómica (se presenta en pacientes con lesión por encima de D5- D6 debida a una desregulación del sistema nervioso vegetativo, provocándole una taquicardia y elevación de la tensión arterial por encima de 250-300 mg de Hg).

Complicaciones urológicas (Vejiga neurógena, infecciones del tracto urinario, litiasis del tracto urinario, reflujo vesicoureteral, fistulas y divertículos) complicaciones gastrointestinales (estreñimiento, incontinencia fecal) deterioro neurológico progresivo, trastornos respiratorios, dolor, trastornos de la reproducción y la sexualidad ${ }^{(1-3)}$

Los efectos de la lesión de la médula espinal sobre la función vesical dependen de la localización de la lesión en la médula espinal. Pueden ocurrir dos tipos de disfunción vesical, vejiga automática y vejiga fláccida.

Vejiga automática es cuando aparece la lesión encima del centro de reflejo de micción en la porción sacra de la médula espinal en T12 o por encima, las personas 
pierden tanto la sensación que necesitan para la micción como el control voluntario, coordinado sobre el centro de micción reflejo. Cuando la vejiga se llena lo suficiente como para que los receptores elásticos en el músculo detrusor se activen, la contracción vesical no controlada, espontánea, se estimula por los simples arcos reflejos. La vejiga automática a menudo no se vacía de manera eficiente. El espasmo del músculo de vaciado vesical (esfínter) puede prevenir la expulsión completa de orina, y derivar en hiperextensión vesical.

Vejiga fláccida está causada por una lesión de la neurona motriz inferior, con daño en el centro reflejo de micción en la porción sacra de la médula espinal. La actividad refleja y la actividad vesical se ven disminuidos y generalmente resultan en una sobre distensión de la vejiga.

Los problemas que provienen de la disfunción del músculo detrusor y del esfínter además de la incontinencia urinaria, pueden incluir infecciones renales recurrentes y presión lumbar alta en los riñones. Finalmente, ambos pueden dar lugar a una grave enfermedad renal.

El tratamiento de la vejiga neurógena, por consiguiente, tiene como objetivo primario mantener la continencia, asegurar una baja presión vesical (para evitar el daño renal) y evitar o disminuir la infección. Las opciones de tratamiento incluyen cateterismo uretral intermitente, cateterismo uretral o suprapúbico permanente, evacuación cronometrada, uso de colector urinario (solo para hombres), cito-plastia de aumento y desviaciones urinarias. ${ }^{(4)}$

Fue en 1844 Stromeyer quien describió por primera vez el sondaje vesical periódico, regular y frecuente para evacuar el volumen residual ${ }^{(5)}$. Durante la Segunda Guerra Mundial se aplicó en el tratamiento de la vejiga neurógena de los adultos con traumatismo medular, pero el método de aplicar el concepto de autosondaje intermitente limpio se debe al urólogo Lapides en la década de los 70, donde mostró la eficiencia e inocuidad a largo plazo de esta modalidad de terapéutica frente a tratamientos utilizados hasta entonces ${ }^{(6)}$.

El sondaje intermitente consiste en pasar una sonda hacia la vejiga para drenar la orina por la uretra u otro canal como la desviación urinaria continente. La sonda se quita inmediatamente después de completar el drenaje de la orina. El sondaje intermitente se puede realizar en personas de todas las edades, incluidos los ancianos y niños de cuatro años de edad con supervisión paterna, también se puede enseñar a los cuidadores a realizar el procedimiento. Las discapacidades como la ceguera, la ausencia de sensación perineal, el temblor, la discapacidad mental y la paraplejia no impiden necesariamente el control de la técnica. ${ }^{(7)}$

Los planes de atención individualizados ayudan a identificar la frecuencia apropiada de sondaje, en base a la exposición de los trastornos urinarios y la repercusión sobre la calidad de vida.

Las ventajas del sondaje intermitente frente al sondaje permanente incluyen:

Mayor oportunidad para el auto cuidado y la independencia de los individuos.

\# Reducción del riesgo de complicaciones comunes asociadas a la sonda permanente. 
\# Reducción de la necesidad de instrumental y artefactos, como bolsa de drenaje.

Mayor libertad de expresión para la sexualidad.

Posibilidad de reducción de los síntomas urinarios (polaquiuria, tenesmo, incontinencia) entre cada intervalo del sondaje. ${ }^{(8)}$

Los tipos y características de las sondas para el sondaje intermitente varían considerablemente, las sondas no recubiertas simples (PVC plástico duro) están cubiertas simplemente con un envase estéril. La mayoría de las sondas se usan con un lubricante separado, aunque es un asunto de elección personal y algunos pacientes no utilizan lubricantes (o solo utilizan agua). La técnica de la limpieza varía con agua y jabón, hervidas, sumergidas en desinfectantes o pasadas por el microondas, las sondas limpiadas se secan y luego guardadas en un recipiente adecuado.

Las sondas recubiertas son de un solo uso (no pueden limpiarse ni reutilizarse). Están diseñadas para mejorar la lubricación de las sondas y la facilidad de la inserción de forma que se pueda reducir los traumatismos y las infecciones de orina.

Los revestimientos más frecuentes son hidrofílicos (que requiere agregar agua a la sonda para formar una capa lubricante) o pre-lubricados (donde la sonda se provee pre-envasada con un revestimiento de gel hidrosoluble) ${ }^{(9-11)}$

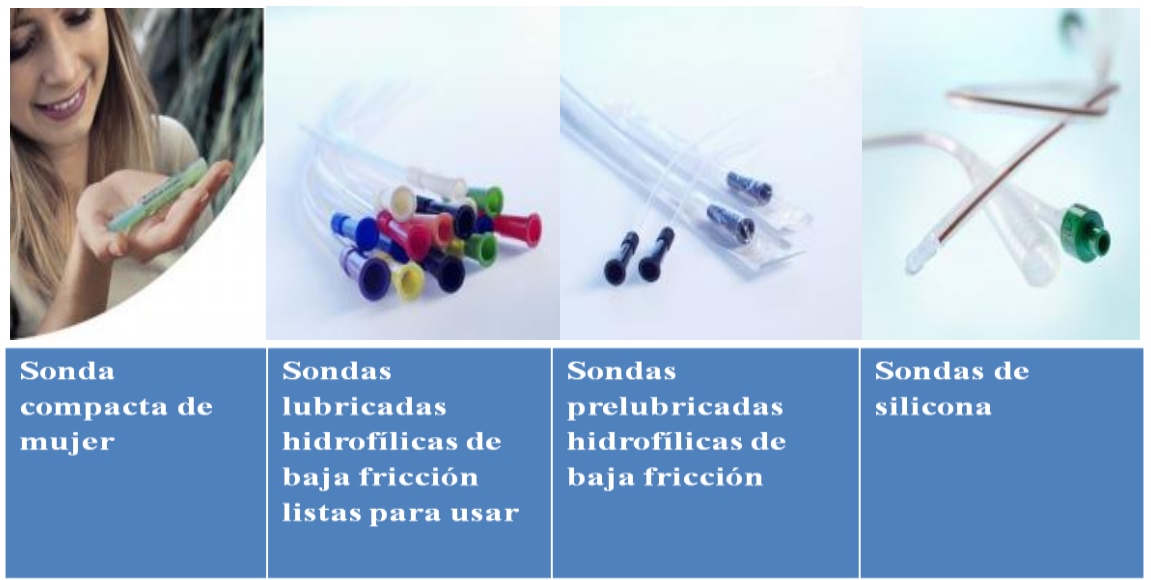

\section{MATERIAL Y METODOS}

La estrategia de búsqueda para la identificación de los artículos, se realizó buscando en bases de datos como CINAHL, PUBMED, BIBLIOTECA VIRTUAL DE LA SALUD, CSIC, BIBLIOTECA COCHRANE PLUS. La fecha de búsqueda de estudios para realizar esta revisión se hizo desde noviembre de 2001 hasta mayo de 2012.

Se llevó a cabo además una búsqueda manual de artículos en revistas especializadas de enfermería tanto españolas como extranjeras desde enero de 2006 hasta mayo de 2012: 


\section{Enfermería Docente}

Enfermería Clínica

Nursing

Enfermería Intensiva

Actas urológicas

Las búsquedas se realizaron mediante el sistema de palabras clave. Se llevó a cabo la búsqueda mediante los descriptores $\mathrm{DeCS} / \mathrm{MeSH}$

Los términos de búsqueda utilizados MesH fueron:

\begin{tabular}{|l|l|l|}
\multicolumn{2}{c}{ TABLA I } \\
\hline Paraplegia & AND & Bacteriuria \\
\hline Urinary incontinence & OR & Nursing assessment \\
\hline Catheter indwelling & OR & Paraplegia \\
\hline Spinal cord injuries & OR & Nursing assessment \\
\hline
\end{tabular}

Los términos de búsqueda utilizados DeCS fueron:

\begin{tabular}{|l|l|l|}
\multicolumn{2}{c}{ TABLA II } \\
\hline Paraplejía & AND & Bacteriuria \\
\hline Incontinencia urinaria & OR & Evaluación en enfermería \\
\hline Catéter de permanencia & OR & Paraplejía \\
\hline Traumatismo de la médula espinal & OR & Evaluación de enfermería \\
\hline
\end{tabular}

Se obtuvieron:

45 artículos en Medline (Pubmed).

5 en Cinahl de los cuales 4 se repetían en Medline.

20 en BVS.

7 en Biblioteca Cochrane Plus.

25 artículos entre las revistas.

Se desecharon varios artículos, unos por no estar dentro de los años de búsqueda y se evaluaron los artículos que se centraron en la incidencia de la infección urinaria en los usuarios de sondajes intermitentes que usaban diferentes técnicas de colocación de la sonda (estéril o limpia); diferentes tipos de sondas (prelubricada o con el lubricante separado); sondas estériles (de uso único) o sondas limpias (de uso múltiple);colocación de la sonda por parte del paciente o por parte de terceros; y otras estrategias para reducir la infección urinaria, incluida la limpieza de las sondas.

\section{RESULTADOS}

No hay estudios definitivos que revelen que la incidencia de la bacteriuria mejore con alguna técnica, estrategia o tipo de sonda, la dificultad de estos estudios consiste pues en que se necesita un periodo largo de tiempo y muchos pacientes abandonan el estudio como explica Moore. ${ }^{(9)}$ 
Harris para bajar el índice de las bacteriurias asociadas a la inserción de sondas vesicales, sugiere crear un protocolo de excelencia para el cuidado urinario, donde solo se va a insertar las sondas vesicales justificadas, se utilizará una técnica aséptica y un cuidado apropiado. (12)

En el estudio observacional y prospectivo de la evaluación de la calidad en pacientes con lesión medular llevado a cabo por Sánchez-Raya no se encuentran diferencias significativas excepto en la dimensión de las limitación de los roles, donde se obtienen mejor puntuación el grupo portador de colectores permanentes que los de sondaje vesical permanente y los cateterismos intermitentes. ${ }^{(13)}$

En el estudio de prevención de las infecciones del tracto urinario relacionadas con las sondas vesicales Dumont refiere las prácticas asistenciales idóneas basadas en la evidencia indican el uso de un sistema de recuerdo para extracción de la sonda vesical, pueden ser de tipo físico o virtual y con estas acciones potenciamos la seguridad del paciente hospitalizado ${ }^{(10)}$

\section{DISCUSIÓN}

Las diferentes técnicas de sondaje y tipos de sondas nos llevan a no poder cuantificar la influencia que tiene en la calidad de vida del lesionado medular y tendríamos que dar respuesta por separado a las siguientes cuestiones:

* El uso de técnica estéril o limpia

El uso de sonda recubierta o no recubierta

- Sonda de un solo uso o uso múltiple

Colocación de la sonda realizada por el mismo paciente o por terceros

Tal como sugiere Moore se necesitan ensayos controlados aleatorios amplios para dar respuesta, estos estudios que son difíciles de realizar. Se sugiere que en el ámbito extra hospitalario la técnica estéril no resulta práctica pero en el ámbito hospitalario, la preocupación creciente acerca del control de infecciones indica que se necesitaría una técnica estéril para mayor seguridad. ${ }^{(9)}$

Se recomienda realizar nuevas investigaciones que tengan en cuenta la relación coste-eficacia, sería importante para llevar a cabo otros estudios de investigación, ya que hay una gran variabilidad de precios entre los distintos tipos de sondas.

La satisfacción, aceptabilidad y preferencia de los pacientes son variables de resultados secundarias que necesitan ser evaluadas en ensayos futuros y estos resultados serian particularmente útiles cuando se evalúa la relación costo-efectividad de los productos de uso único/reutilizable. ${ }^{(9)}$

\section{CONCLUSIONES}

No hay pruebas suficientes para demostrar la efectividad de un tipo de sonda, técnica o estrategia en particular. Las variaciones en la práctica clínica y el crecimiento de la utilización de las sondas de uso único, con mayores costos asociados, muestra que se necesita un ensayo controlado aleatorio bien diseñado.

La retirada temprana de las sondas vesicales permanentes se asocia con un menor riesgo de infección del tracto urinario y una estancia más corta en el hospital. Las particularidades clínicas de estas infecciones en pacientes con vejiga neurogénica 
por lesión de la médula espinal necesitan adecuadas estrategias para el manejo clínico, microbiológico y epidemiológico de las mismas.

El objetivo principal es determinar si ciertos tipos de sondas y técnicas de colocación de la sonda son mejores para la prevención de complicaciones a largo plazo en la incontinencia urinaria y como objetivos secundarios serían: conocer la influencia en la calidad de vida, la reducción de infecciones urinarias y la relación costo-efectividad en el tratamiento vesical a largo plazo.

\section{BIBLIOGRAFÍA}

1. Romero-Ganuza F.J, Mazaira-Álvarez J. El paciente con lesión medular en el medio extra hospitalario. Aten Primaria. 2001; 27(2): 127-36

2. Vincent M, Vacca, Jr. Disreflexia autonómica. Nursing (Barc).2008; 26(7):7.

3. Donna A. Nayduch, Los fundamentos, identificación y tratamiento de la lesión medular. Nursing (Barc).2011; 29(3):8-15.

4. The Cochrane Database, Issue 2, 2008. [base de datos de internet].Oxfort: Update Software Ltd; 1998-[fecha de consulta 25 de octubre de 2011].Jamison J, Maguire S, McCann J. Políticas del uso de catéteres para el tratamiento de problemas de micción a largo plazo en pacientes adultos con trastornos vesicales neurogénicos. (Cochrane Review). Disponible en:http//www.updatesoftware.com./Cochrane/.

5. Smith PF. German orthopaedic and military surgeon and his links with Britain. $\underline{\mathrm{J}}$ Med Biogr. 2006; 14(2):65-74.

6. Wyndaele JJ. Complications of intermittent catheterization: their prevention and treatment. Spinal Cord. 2002;40(10):536-41

7. Arlandis-Guzmán S, Ruiz-Cerdá J.L, Martínez-Agullo E. Las pruebas funcionales urodinámicas en el diagnostico de la vejiga neurógena. Rehabilitación (Madr) 2005; 39(6):343-57

8. Sánchez-Villar I. Retirada de la sonda vesical permanente de corta duración. Enferm Clin.2008; 18(2):107-8.

9. The Cochrane Database, Issue 3, 2008. [base de datos de internet].Oxfort: Update Software Ltd; 1998-[fecha de consulta 25 de octubre de 2011].Moore KN, Fader M, Getliffe K. Tratamiento vesical a largo plazo con sondaje intermitente en adultos y niños. . (Cochrane Review). Disponible en: http//www.update-software.com./Cochrane/.

10. Dumont Ch, Wakeman J. Prevención de las infecciones del tracto urinario relacionado con las sondas vesicales: resultados de un estudio. Nursing (Barc).2011; 29:30-6.

11.Sánchez I. Retirada de la sonda vesical permanente de corta duración. Enferm Clin.2008; 18(2):107-8.

12. Harris T. Variar la práctica para reducir la utilización de las sondas vesicales. Nursing( Barc).2010;28(7):48-9

13. Sánchez-Raya J, Romero-Culleres G, González Viejo M.A, Ramírez Garcerán L, García Fernández L, Conejero Sugrañes J. Evaluación de la calidad de vida en pacientes con lesión medular comparando diferentes métodos de vaciado vesical. Actas Urol Esp.2010;34(6):537-42 


\section{ANEXO I}

CUESTIONARIO King's Health. Evaluación de la calidad de vida en pacientes con lesión medular comparando diferentes métodos de vaciado vesical.

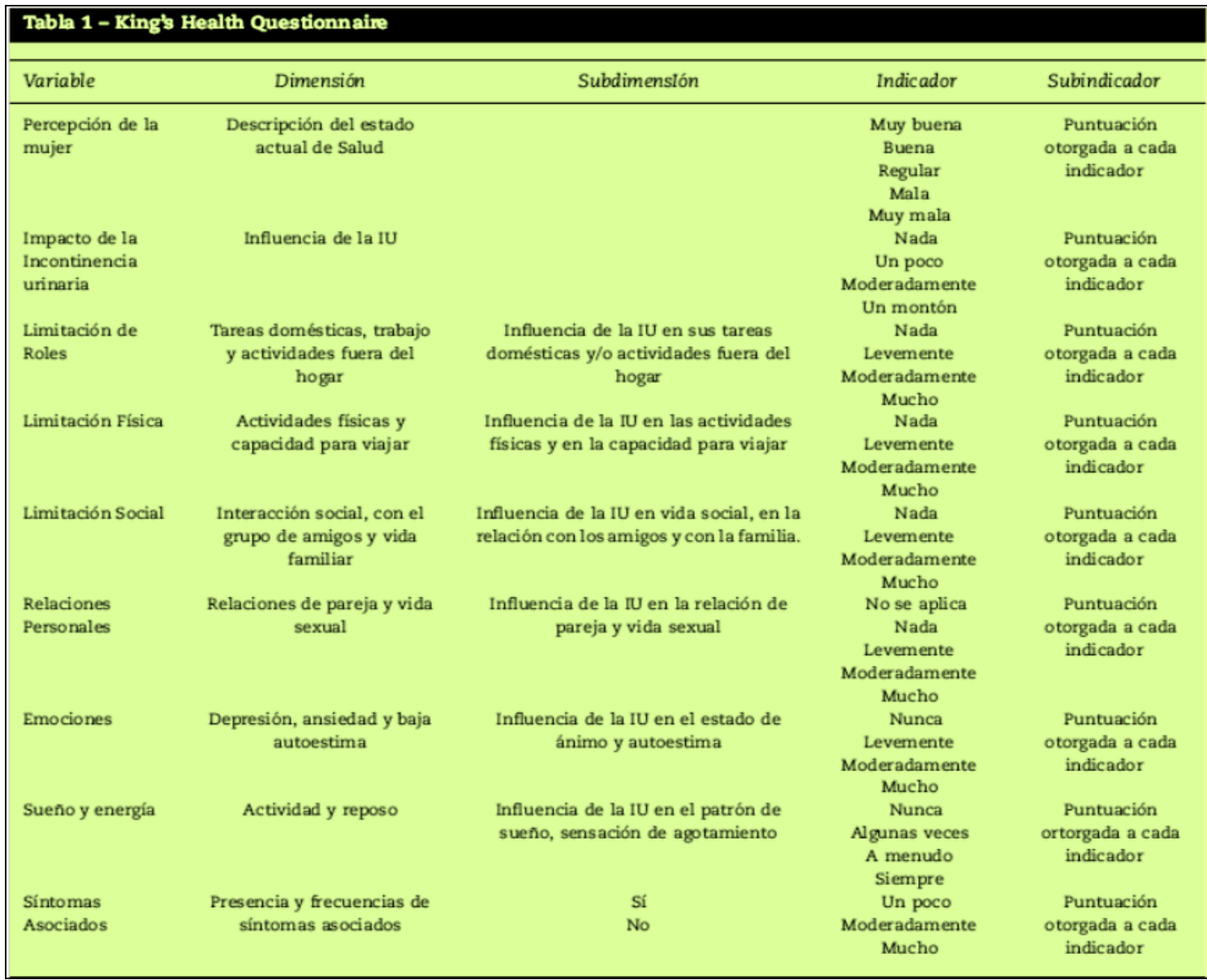

\section{ANEXO II}

Cuestionario King's Health. Evaluación de la calidad de vida en pacientes con lesión medular comparando diferentes métodos de vaciado vesical.

\section{Tabla 2 - Problemas relacionados con la bsión mechlar}

\begin{tabular}{l} 
Problemas \\
$\begin{array}{c}\text { Ordenar de mayor a } \\
\text { menor (1-9) }\end{array}$ \\
\hline Problemas de deambulación \\
Tramites administrativos \\
Uso de trasportes públicos \\
Disfunción sexual \\
Problemas con la defecación \\
Problemas urinarios \\
Úlceras \\
Prejuicios con la gente \\
Otros
\end{tabular}


๑ COPYRIGHT Servicio de Publicaciones - Universidad de Murcia 